\title{
Clinical condition and comorbidity as determinants for blood culture positivity in patients with skin and soft-tissue infections
}

\author{
F. V. van Daalen ${ }^{1}$ M. C. Kallen ${ }^{1}$ - C. M. A. van den Bosch ${ }^{1}$ - M. E. J. L. Hulscher ${ }^{2}$. \\ S. E. Geerlings ${ }^{1}$ • J. M. Prins ${ }^{1}$
}

Received: 22 March 2017 / Accepted: 24 April 2017 / Published online: 7 June 2017

(C) The Author(s) 2017. This article is an open access publication

\begin{abstract}
The utility of performing blood cultures in patients with a suspected skin infection is debated. We investigated the association between blood culture positivity rates and patients' clinical condition, including acute disease severity and comorbidity. We performed a retrospective study, including patients with cellulitis and wound infection who had been enrolled in three Dutch multicenter studies between 2011 and 2015. Patients' acute clinical condition was assessed using the Modified Early Warning Score (MEWS; severe: MEWS $\geq 2$ ) and comorbidity with the Charlson Comorbidity Index (CCI; severe: $\mathrm{CCI} \geq 2$ ). A total of 334 patients with a suspected skin infection were included. Blood cultures were performed in 175 patients (52\%), 28 of whom (16\%) had a positive blood culture. Data on the clinical condition were collected in 275 patients. Blood cultures were performed in $76 \%$ of the patients with a severe acute condition, compared with $48 \%$ with a non-severe acute condition (OR 3.5; 95\% confidence interval: 2.0-6.2; $p<0.001$ ). Blood cultures were positive in $18 \%$ and $12 \%$ respectively (OR $1.7(0.7-4.1) ; p=0.3)$. Blood cultures were performed in $53 \%$ of patients with severe comorbidity, compared with $61 \%$ without severe comorbidity (OR 0.7 ;
\end{abstract}

Frederike V. van Daalen and Marlot C. Kallen contributed equally to this article.

F. V. van Daalen

f.v.vandaalen@amc.uva.nl

1 Department of Internal Medicine, Division of Infectious Diseases, Academic Medical Centre, University of Amsterdam, Room F4-132, Meibergdreef 9, 1105 AZ Amsterdam, the Netherlands

2 Department of Scientific Institute for Quality of Healthcare (IQ healthcare), Radboud University Medical Center, Nijmegen, the Netherlands
$0.4-1.2 ; p=0.2)$. Blood cultures were positive in $25 \%$ and $10 \%$ respectively $(\mathrm{OR}=3.1 ; 1.2-7.5 ; p=0.02)$. The blood culture positivity rate among hospitalized patients diagnosed with skin infections was higher than the rates reported by the Infectious Diseases Society of America guidelines, particularly in patients with severe comorbidity. Therefore, the recommendations concerning blood culture performance in patients with a skin infection should be reconsidered.

\section{Introduction}

Acute bacterial skin and soft-tissue infections (SSTI) are among the most common indications for antibiotic use in hospitalized adults [1]. Cellulitis involves the deep dermis and subcutaneous tissues, whereas erysipelas is limited to superficial dermal structures with well-defined borders, although it is presently considered a manifestation of cellulitis [2-5]. Most of these infections arise from beta-hemolytic streptococci. Staphylococcus aureus can cause cellulitis, typically in the presence of an open wound or previous penetrating trauma. Several other organisms can also cause cellulitis, but usually only in special circumstances [2]. Wound infections are defined by the presence of a preexisting skin lesions at the time of skin infection onset, such as a chronic ulcer or a trauma-related skin lesion, and can vary from superficial skin infection to deeper infection with involvement of tissues under the skin [6,7].

The current Infectious Diseases Society of America (IDSA) guidelines do not recommend routine performance of blood cultures in patients with cellulitis and erysipelas [2]. Previous studies reported low positivity rates (4-13\%) and marginal consequences for treatment, as standard empirical therapy covers most causative pathogens, including betahemolytic streptococci $[2,3,8-11]$. 
Although $S$. aureus and gram-negative bacteria are less frequently noted to cause SSTI, they require different treatment $[2,9]$. As these pathogens are more frequently found in specific patient groups, IDSA guidelines recommend performing blood cultures only in patients with malignancy, chemotherapy, neutropenia, severe cell-mediated immunodeficiency, immersion injuries, and animal bites [2]. These recommendations, however, are based on little evidence and do not take the patients' acute clinical condition into account.

The aim of our study was to investigate the association between blood culture positivity rates and the patient's clinical condition, including acute disease severity and comorbidity.

\section{Materials and methods}

\section{Study setting and population}

We performed a retrospective study in the databases of two multicenter observational studies (RIANT and IMPACT) and one multicenter intervention study ( $\mathrm{AB}$ checklist), performed between 2011 and 2015 in 40 university and non-university hospitals in the Netherlands (http://www.trialregister.nl; NTR 5933) $[12,13]$. The RIANT study developed and validated a set of generic quality indicators to define and measure the appropriateness of antibiotic use in the treatment of bacterial infections in hospitalized adult patients. The IMPACT study compares different methods, including a point prevalence survey using these validated quality indicators, to measure the appropriateness of antibacterial use in hospitalized adult patients. The $\mathrm{AB}$ checklist study implemented an antibiotic checklist based on these validated quality indicators and evaluated the effect of checklist use on the length of hospital stay and appropriate antibiotic use. Inclusion criteria for all three studies were: adult patients ( $\geq 18$ years old) admitted to an acute care department with a suspected bacterial infection and treated with antibiotics (ATC group J01) for at least 24 hours. In all three studies, patients were excluded if antibiotics were used as prophylaxis, or when antibiotic treatment was started on the ICU department or in another hospital. All patients were randomly selected. For the present study, we identified the patients with cellulitis in these databases, including those with a clinical diagnosis of erysipelas; we also included patients with a wound infection.

\section{Assessments}

Data were collected from electronic medical records and medication charts. In all three studies, data were recorded on patient characteristics, including sex, age and diagnosis, and whether blood culture had been performed, including the number of cultures and their results.
In two studies, RIANT and $\mathrm{AB}$ checklist, additional data were recorded on the acute clinical condition and comorbidity $[12,13]$. Patients' acute clinical condition was assessed at the start of treatment using the Modified Early Warning Score (MEWS) [14]. A severe acute condition was defined by MEWS $\geq 2$, non-severe acute condition by MEWS $<2$. Comorbidity was assessed using the Charlson Comorbidity Index (CCI) [15] and severe comorbidity was defined by $\mathrm{CCI} \geq 2$.

\section{Analysis}

Numbers of blood cultures taken and numbers of positive cultures, including type of pathogen, were combined for all three studies and expressed in absolute numbers and percentages. We compared the proportions of positive blood cultures between patients diagnosed with cellulitis, those with a clinical diagnosis of erysipelas, and those with a wound infection. In the evaluation of the association between blood culture positivity rates and patients' clinical condition we only included patients of the RIANT and AB checklist studies $[12,13]$. We compared the proportions of patients with a blood culture taken and positivity rates between patients with a severe and those with a non-severe acute clinical condition, and between patients with and without severe comorbidity. Additionally, we performed a sub-analysis in which we compared patients with or without diabetes mellitus. We used Chisquared tests to compare proportions. As age and comorbidity are often correlated, we performed a multivariate logistic regression analysis to investigate the association between the positivity rate of blood cultures and acute clinical condition, comorbidity and age of the patient. $P<0.05$ was considered statistically significant. Analyses were carried out using IBM SPSS Statistics, version 23.0.

\section{Results}

A total of 334 patients with a suspected SSTI were included in our study, of whom 258 (77\%) were diagnosed with cellulitis and $76(23 \%)$ with a wound infection. Blood cultures were performed in 175 out of 334 patients (52\%): in 153 out of 258 patients $(59 \%)$ with cellulitis and in 22 out of 76 patients (29\%) with a wound infection. Twenty-eight out of 175 patients $(16 \%)$ had a positive blood culture: 23 out of 153 patients $(15 \%)$ with cellulitis and 5 out of 22 with a wound infection (23\%). One hundred and nine of the patients with cellulitis $(42 \%)$ had a clinical diagnosis of erysipelas. Blood cultures were performed in 70 out of 109 patients (64\%) with erysipelas and 8 out of $70(11 \%)$ had a positive blood culture. Table 1 presents the baseline characteristics of all patients, of patients in whom blood cultures were performed, and of patients with positive blood cultures. 
Table 1 Baseline characteristics of patients with a suspected skin and soft-tissue infection (SSTI)

\begin{tabular}{llll}
\hline Characteristics $^{\text {a }}$ & All patients & $\begin{array}{l}\text { Patients in whom blood } \\
\text { cultures were performed } \\
n=175\end{array}$ & $\begin{array}{l}\text { Patients with positive } \\
\text { blood cultures } \\
n=28\end{array}$ \\
\hline $\begin{array}{l}\text { Sex, male } \\
\text { Age, mean (SD) }\end{array}$ & $181(55)$ & $96(55)$ & $15(54)$ \\
Hospital & $66.0(17)$ & $65.1(16)$ & $69.7(16)$ \\
$\quad$ University & $42(13)$ & $17(10)$ & $3(11)$ \\
$\quad$ Non-university & $292(87)$ & $158(90)$ & $25(89)$ \\
Diagnosis & & & $23(82)$ \\
Cellulitis & $258(77)$ & $153(87)$ & $8(29)$ \\
$\quad$ Erysipelas subgroup & $109(33)$ & $70(40)$ & $5(18)$ \\
$\quad$ Wound infection & $76(23)$ & $22(13)$ & $28(100)$ \\
Antibiotics & & $172(98)$ & $0(0)$ \\
Started intravenously (IV) & $312(93)$ & $3(2)$ & $28(100)$ \\
$\quad$ Started orally (O) & $22(7)$ & $175(100)$ & \\
Total & $334(100)$ & &
\end{tabular}

${ }^{\text {a }}$ Numbers are $n(\%)$, unless otherwise indicated
No significant difference was found in positivity rates between the patients with a diagnosis of cellulitis not classified as erysipelas $(n=83)$ and those with a clinical diagnosis of erysipelas $(n=70$; OR 0.6 ; $95 \%$ confidence interval: $0.2-1.5$; $p=0.3)$, or between patients with cellulitis $(n=153)$ and those with a wound infection (OR $0.6 ; 0.2-1.8 ; p=0.4$ ).

Twenty-nine pathogens were identified. Most cultured species were Streptococcus species (in 12 cultures), Gramnegative bacteria (in 8 cultures), and Staphylococcus aureus (in 7 cultures). See Table 2 for details per diagnosis.

Data on acute clinical condition and comorbidity were recorded in 275 patients, of whom 214 (78\%) were diagnosed with cellulitis and $61(22 \%)$ with a wound infection. Eightyfive out of $275(31 \%)$ had a severe acute clinical condition, 102 out of 275 (37\%) had severe comorbidity. Table 3 presents the baseline characteristics of these patients. Blood cultures were performed in $76 \%$ of the patients with a severe acute clinical condition, compared with $48 \%$ of patients with a non-severe condition (OR 3.5; 1.9-6.2; $p<0.001$ ). Blood cultures were positive in $18 \%$ and $12 \%$ respectively (OR 1.7 ; $0.7-4.1 ; p=0.3)$. In $53 \%$ of the patients with a severe comorbidity blood cultures were performed, compared with $61 \%$ of patients without a severe comorbidity $(\mathrm{OR}=0.7 ; 0.4-1.2$; $p=0.2$ ). Blood cultures were positive in $25 \%$ and $10 \%$ respectively $(\mathrm{OR}=3.1 ; 1.2-7.5 ; p=0.02$; Table 4$)$.

Of the 275 patients included in the comorbidity analysis, 73 were diagnosed with diabetes mellitus. Blood cultures were performed in 44 of these patients. Blood cultures were positive in 9 out of 44 of the patients with diabetes (20\%), compared with 14 out of $113(13 \%)$ of the patients without diabetes (OR $1.8 ; 0.7-4.6 ; p=0.2$ ).

The mean age of patients with severe comorbidity was higher than the mean age of patients without severe comorbidity
(Table 4). A higher age was significantly associated with positive blood cultures $(\mathrm{OR}=1.03 ; 1.0-1.06 ; p=0.07)$. In the multivariate regression analysis, the severity of comorbidity was a stronger predictor of positive blood cultures than age (CCI: OR 2.3; 0.9-6.2; $p=0.09$, compared with age: OR 1.0; $1.0-1.1 ; p=0.3)$.

\section{Discussion}

In this study, we found that blood cultures were taken in 52\% of patients hospitalized with cellulitis or wound infections, with a positivity rate of $16 \%$. Significantly more blood cultures were performed in patients with a severe compared with patients with a non-severe acute clinical condition, but positivity rates of blood cultures were significantly higher in patients with severe comorbidity.

The positivity rate in our study was higher than in previous studies, where blood cultures were found to be positive in 4 $9 \%$ of patients with cellulitis or erysipelas $[2,8,9,11]$. In one study, higher positivity rates were found for purulent compared with nonpurulent infections [7]. Definitions and inclusion criteria differed between studies, which may explain the range in positivity rates.

Our study supports previous findings stating that Streptococci species are the most frequently cultured species in patients with cellulitis and erysipelas, followed by $S$. aureus and Gramnegative bacteria [9]. S. aureus was cultured in 3 of the 5 patients with a wound infection and a positive blood culture, in line with the literature describing that this pathogen is typically found in patients with an open wound or previous penetrating trauma [2]. Remarkably, in 4 out of 8 patients with a clinical diagnosis of 
Table 2 Microbiology results of patients with a suspected SSTI and a positive blood culture

\begin{tabular}{llll}
\hline Pathogen & $\begin{array}{l}\text { Total number } \\
\text { of pathogens }\end{array}$ & Number of pathogens per diagnosis & \\
\cline { 3 - 4 } & Cellulitis & Wound infection
\end{tabular}

Erysipelas subgroup

\begin{tabular}{|c|c|c|c|c|}
\hline \multicolumn{5}{|l|}{ Negative culture results } \\
\hline No pathogen & $140(96)$ & 125 & 59 & 15 \\
\hline Contamination & $6(4)$ & 5 & 3 & 1 \\
\hline Total & $146(100)$ & 130 & 62 & 16 \\
\hline \multicolumn{5}{|l|}{ Positive culture results } \\
\hline \multicolumn{5}{|l|}{ Staphylococcus species } \\
\hline S. aureus & $7(24)$ & 4 & & 3 \\
\hline S. capitis & $1(3)$ & 1 & 1 & \\
\hline \multicolumn{5}{|l|}{ Streptococcus species } \\
\hline S. pneumoniae & $1(3)$ & 1 & & \\
\hline S. pyogenes & $3(10)$ & 3 & 1 & \\
\hline S. dysgalactiae & $2(7)$ & 2 & 1 & \\
\hline S. salivarius & $1(3)$ & & & 1 \\
\hline Group A & $1(3)$ & 1 & & \\
\hline Group B & $2(7)$ & 2 & & \\
\hline Group C & $1(3)$ & 1 & & \\
\hline Group G & $1(3)$ & 1 & 1 & \\
\hline \multicolumn{5}{|l|}{ Enterococcus species } \\
\hline E. faecium & $1(3)$ & 1 & & \\
\hline \multicolumn{5}{|l|}{ Gram-negative bacteria } \\
\hline E. coli & $1(3)$ & & & 1 \\
\hline K. oxytoca & $2(7)$ & 2 & 1 & \\
\hline P. aeruginosa & $3(10)$ & 3 & 1 & \\
\hline Pseudomonas species & $1(3)$ & 1 & 1 & \\
\hline H. influenza & $1(3)$ & 1 & 1 & \\
\hline Total & $29^{\mathrm{b}}(100)$ & 24 & 8 & 5 \\
\hline
\end{tabular}

${ }^{\text {a }}$ Numbers are $n(\%)$, unless otherwise indicated

${ }^{\mathrm{b}}$ In one patient two pathogens were identified erysipelas and a positive culture, Gram-negative bacteria were cultured.

Clinicians were more likely to perform blood cultures in patients with a severe acute clinical condition, defined by a MEWS $\geq 2$. A recent Swedish study described a similar association, showing that blood cultures were more frequently performed in patients who fulfilled the severe inflammatory response syndrome (SIRS) criteria [8]. However, they did not compare the positivity rates of blood cultures between patients who did or did not fulfill the SIRS criteria. An American retrospective chart review did look at positivity rates and acute clinical condition: they compared - among others-positivity rates of blood cultures in patients with cellulitis with or without fever [11]. Unexpectedly, patients without fever had positive blood cultures significantly more frequently than patients with fever, which supports our findings that a severe acute clinical condition is not associated with a higher positivity rate.
In our study clinicians were not more likely to perform blood cultures in patients with severe comorbidity, defined by a $\mathrm{CCI} \geq 2$, but the positivity rate was significantly higher in these patients. An association between comorbidity and positivity rates has been described before, especially for malignancy, immunodeficiency or diabetes mellitus $[2,10]$. Diabetes mellitus is a comorbidity with a high prevalence and often contributes to the total CCI score. A relationship between diabetes mellitus and positive blood cultures has been described [16]. In our study, the positivity rate for patients with diabetes was also higher than in patients without diabetes, but this difference was not statistically significant. As age is the most important risk factor for the presence of comorbidities, an association between the patient's age and the positivity rate of blood cultures is plausible. The multivariate regression analysis illustrated, however, that comorbidity is a better predictor of blood culture positivity. 
Table 3 Baseline characteristics of 275 patients in the clinical condition analyses

\begin{tabular}{|c|c|c|c|c|}
\hline \multirow[t]{2}{*}{ Characteristics $^{\mathrm{a}}$} & \multicolumn{2}{|c|}{$\begin{array}{l}\text { Patients included in the acute } \\
\text { clinical condition analysis }\end{array}$} & \multicolumn{2}{|c|}{$\begin{array}{l}\text { Patients included in the } \\
\text { comorbidity analysis }\end{array}$} \\
\hline & $\begin{array}{l}\text { MEWS <2 } \\
n=190\end{array}$ & $\begin{array}{l}\text { MEWS } \geq 2 \\
n=85\end{array}$ & $\begin{array}{l}\text { CCI }<2 \\
n=173\end{array}$ & $\begin{array}{l}\mathrm{CCI} \geq 2 \\
n=102\end{array}$ \\
\hline Sex, male & $112(59)$ & $36(42)$ & $102(59)$ & $46(45)$ \\
\hline Age, mean (SD) & $66.1(17)$ & $66.2(17)$ & $61.6(17)$ & $73.8(14)$ \\
\hline \multicolumn{5}{|l|}{ Hospital } \\
\hline University & $19(10)$ & $10(12)$ & $15(9)$ & $14(14)$ \\
\hline Non-university & $171(90)$ & $75(88)$ & $158(91)$ & $88(86)$ \\
\hline \multicolumn{5}{|l|}{ Antibiotic at start of treatment } \\
\hline Flucloxacillin & $98(52)$ & $40(47)$ & $99(57)$ & $39(38)$ \\
\hline Amoxicillin-clavulanic acid & $39(21)$ & $18(21)$ & $33(19)$ & $24(23)$ \\
\hline Clindamycin & $17(9)$ & $3(4)$ & $10(6)$ & $10(10)$ \\
\hline Penicillin & $10(5)$ & $8(9)$ & $12(7)$ & $6(6)$ \\
\hline Cefuroxime & $6(3)$ & $3(4)$ & $6(3)$ & $3(3)$ \\
\hline Ceftriaxone & $2(1)$ & $2(2)$ & $1(1)$ & $3(3)$ \\
\hline Other & $18(9)$ & $11(13)$ & $12(7)$ & $17(17)$ \\
\hline \multicolumn{5}{|l|}{ IV / oral } \\
\hline Started intravenously (IV) & $179(94)$ & $79(93)$ & $169(98)$ & $89(87)$ \\
\hline Started orally $(\mathrm{O})$ & $11(6)$ & $6(7)$ & $4(2)$ & $13(13)$ \\
\hline Length of stay, days, geometric mean $(95 \% \mathrm{CI})$ & $7.0(6.2-7.9)$ & $8.3(6.8-10.2)$ & $5.9(5.2-6.6)$ & $10.9(9.1-13.0)$ \\
\hline In-hospital mortality rate & $6(3)$ & $2(2)$ & $2(1)$ & $6(6)$ \\
\hline
\end{tabular}

MEWS Modified Early Warning Score, CCI Charlson Comorbidity Index

${ }^{\text {a }}$ Numbers are $n(\%)$, unless otherwise indicated
Our study has several strengths. We provide recent data on blood culture positivity rates in patients hospitalized with an SSTI, which is necessary as the IDSA guidelines still report positivity rates $\leq 5 \%$ based on a study from 1999 [10]. Our

Table 4 The MEWS and CCI of patients in the clinical condition analysis

\begin{tabular}{|c|c|c|c|}
\hline & $\begin{array}{l}\text { Total } \\
\text { number } \\
\text { of } \\
\text { patients }\end{array}$ & $\begin{array}{l}\text { Number of patients } \\
\text { in whom blood } \\
\text { cultures were } \\
\text { performed }^{\mathrm{a}}\end{array}$ & $\begin{array}{l}\text { Number of } \\
\text { patients with } \\
\text { positive blood } \\
\text { cultures }^{\text {a }}\end{array}$ \\
\hline \multicolumn{4}{|l|}{ MEWS } \\
\hline Non-severe $(<2)$ & 190 & $92(48)$ & $11(12)$ \\
\hline Severe $(\geq 2)$ & 85 & $65(76)$ & $12(18)$ \\
\hline Total & 275 & $157(57)$ & $23(15)$ \\
\hline $\begin{array}{l}\text { Odds ratio, } 95 \% \\
\text { confidence interval, } p\end{array}$ & $p$ value & $\begin{array}{r}3.5,2.0-6.2 \\
\quad p<0.001^{\mathrm{b}}\end{array}$ & $\begin{array}{c}1.7,0.7-4.1 \\
p=0.3^{\mathrm{b}}\end{array}$ \\
\hline \multicolumn{4}{|c|}{$\mathrm{CCI}$} \\
\hline Non-severe $(<2)$ & 173 & $104(60)$ & $10(6)$ \\
\hline Severe $(\geq 2)$ & 102 & $53(52)$ & $13(13)$ \\
\hline Total & 275 & $157(57)$ & $23(15)$ \\
\hline $\begin{array}{l}\text { Odds ratio, } 95 \% \\
\text { confidence interval, } p\end{array}$ & $p$ value & $\begin{array}{l}0.7,0.4-1.2 \\
p=0.2^{\mathrm{c}}\end{array}$ & $\begin{array}{l}3.1,1.2-7.5 \\
\quad p=0.02^{\mathrm{c}}\end{array}$ \\
\hline
\end{tabular}

study is large and representative, as we included patients from nearly half of all Dutch hospitals, including university, teaching, and general hospitals.

Our study has limitations. As it was based on retrospective chart reviews, it was the clinicians' decision as regards which patients' blood cultures were performed. Our data clearly show that clinicians are more inclined to perform blood cultures in severely ill patients. However, we also showed that positivity rates were better predicted by comorbidity. Furthermore, because of this retrospective setup, we selected patients based on the diagnosis documented in the patient files and we were dependent on the documentation of health care providers.

In conclusion, the blood culture positivity rate among hospitalized patients diagnosed with SSTIs was higher than the rates reported by the IDSA guidelines, particularly in patients with severe comorbidity. Therefore, the recommendations concerning blood culture performance in patients with an SSTI should be reconsidered.

Acknowledgements All participating hospitals: Academic Medical Center, Amsterdam; Amphia Hospital, Breda; Amstelland Hospital, Amstelveen; Antonius Hospital, Nieuwegein; Atrium Medical Center Parkstad, Heerlen; Bronovo Hospital, Den Haag; Catharina Hospital, Eindhoven; Diakonessenhuis, Utrecht; Diakonessenhuis, Zeist; Erasmus Medical Center, Rotterdam; Flevo Hospital, Almere; Franciscus Hospital, Roosendaal, Gelderse Vallei, Ede; Gelre Hospital, Apeldoorn; Gemini 
Hospital, Den Helder; Groene Hart Hospital, Gouda; Hospital Rivierenland, Tiel; Jeroen Bosch Hospital, Den Bosch; LangeLand Hospital, Zoetermeer; Leiden University Medical Center, Leiden; Lievensberg Hospital, Bergen op Zoom; Maasstad Hospital, Rotterdam; Maastricht University Medical Center, Maastricht; Maxima Medical Center, Veldhoven; Medical Center Haaglanden, Leidsendam, Den Haag; Medical Center Haaglanden, Westeinde, Den Haag; Medisch Spectrum Twente, Enschede; Onze Lieve Vrouw Gasthuis, Amsterdam; Orbis Medical Center, Sittard; Radboud University Medical Center, Nijmegen; Reinier de Graaf Hospital, Delft; Rijnstate Hospital, Arnhem; Spaarnegasthuis, Haarlem; Spaarnegasthuis, Hoofddorp; University Medical Center Groningen, Groningen; University Medical Center Utrecht, Utrecht; Viecuri Hospital, Venlo; Vlietland Hospital, Schiedam; VU University Medical Center, Amsterdam; Westfriesgasthuis, Hoorn.

\section{Compliance with ethical standards}

Funding No funds were received for this study.

The RIANT study was supported by the Netherlands Organization for Health Research and Development (ZonMW), grant 205,100,003. The IMPACT study was supported by the Netherlands Organization for Health Research and Development (ZonMW), grant 205,300,002. The AB checklist study was supported by the Netherlands Organization for Health Research and Development (ZonMW), grant 836,021,001.

Conflicts of interest The authors declare that they have no conflicts of interest.

Ethical approval For this type of study formal consent is not required.

The RIANT study, the IMPACT study and the AB-checklist study were all discussed with the Medical Ethics Research Committee of the Academic Medical Centre. They confirmed that the Medical Research Involving Human Subjects Acts did not apply to these studies, and that official approval by the committee was not required, because patients received treatment according to the standard of care.

Informed consent Not applicable since the study was non-interventional and data were retrospectively analyzed.

Data availability All data analyzed during the current study are included in the published article or available from the corresponding author on reasonable request.

Open Access This article is distributed under the terms of the Creative Commons Attribution 4.0 International License (http:// creativecommons.org/licenses/by/4.0/), which permits unrestricted use, distribution, and reproduction in any medium, provided you give appropriate credit to the original author(s) and the source, provide a link to the Creative Commons license, and indicate if changes were made.

\section{References}

1. Magill SS, Edwards JR, Beldavs ZG et al (2014) Prevalence of antimicrobial use in US acute care hospitals, May-September 2011. JAMA 312(14):1438-1446

2. Stevens DL, Bisno AL, Chambers HF et al (2014) Practice guidelines for the diagnosis and management of skin and soft tissue infections: 2014 update by the Infectious Diseases Society of America. Clin Infect Dis 59(2):e10-e52

3. Raff AB, Kroshinsky D (2016) Cellulitis: a review. JAMA 316(3): 325-337

4. Swartz MN (2004) Clinical practice. Cellulitis. N Engl J Med 350(9):904-912

5. Bisno AL, Stevens DL (1996) Streptococcal infections of skin and soft tissues. N Engl J Med 334(4):240-245

6. Centers for Disease Control and Prevention (2012) Surgical site infection. http://www.cdc.gov/hai/ssi/ssi.html

7. Lee CY, Tsai HC, Kunin CM, Lee SS, Chen YS (2015) Clinical and microbiological characteristics of purulent and non-purulent cellulitis in hospitalized Taiwanese adults in the era of communityassociated methicillin-resistant Staphylococcus aureus. BMC Infect Dis 15:311

8. Blackberg A, Trell K, Rasmussen M (2015) Erysipelas, a large retrospective study of aetiology and clinical presentation. BMC Infect Dis 15:402

9. Gunderson CG, Martinello RA (2012) A systematic review of bacteremias in cellulitis and erysipelas. J Inf Secur 64(2):148-155

10. Perl B, Gottehrer NP, Raveh D, Schlesinger Y, Rudensky B, Yinnon AM (1999) Cost-effectiveness of blood cultures for adult patients with cellulitis. Clin Infect Dis 29(6):1483-1488

11. Paolo WF, Poreda AR, Grant W, Scordino D, Wojcik S (2013) Blood culture results do not affect treatment in complicated cellulitis. J Emerg Med 45(2):163-167

12. Van den Bosch CM, Hulscher ME, Natsch S et al (2016) Applicability of generic quality indicators for appropriate antibiotic use in daily hospital practice: a cross-sectional point-prevalence multicenter study. Clin Microbiol Infect 22(10):888.e1-888.e9

13. Van Daalen FV, Prins JM, Opmeer BC, Boermeester MA, Visser CE, van Hest RM et al (2017) Effect of an antibiotic checklist on length of hospital stay and appropriate antibiotic use in adult patients treated with intravenous antibiotics: a stepped wedge cluster randomized trial. Clin Microbiol Infect 10.1016/j.cmi.2017.01.019

14. Quan H, Sundararajan V, Halfon P et al (2005) Coding algorithms for defining comorbidities in ICD-9-CM and ICD-10 administrative data. Med Care 43(11):1130-1139

15. Subbe CP, Kruger M, Rutherford P, Gemmel L (2001) Validation of a modified early warning score in medical admissions. QJM 94(10): $521-526$

16. Kielhofner MA, Brown B, Dall L (1988) Influence of underlying disease process on the utility of cellulitis needle aspirates. Arch Intern Med 148(11):2451-2452 